\title{
Diversity and Seasonal Availability of Potential Wild Edible Plants from Vidarbha Region of Maharashtra State, India
}

\author{
K.C. Kiran ${ }^{1 *}$, C. Dhanush ${ }^{1}$, C.V Gajendra ${ }^{2}$ and B.M. Reddy ${ }^{3}$ \\ ${ }^{1}$ Department of forestry and environmental science, UAS, Bengaluru, Karnataka, India \\ ${ }^{2}$ Forest collage and research institute, Mettupalayam, TNAU, Tamil Nadu, India \\ ${ }^{3}$ Forest research institute, Dehradun, Uttarkhand, India \\ *Corresponding author
}

\section{A B S T R A C T}

\begin{tabular}{|l|}
\hline Ke y w or d s \\
$\begin{array}{l}\text { Wild edible plants, } \\
\text { Diversity, Food } \\
\text { security, Vidarbha } \\
\text { region }\end{array}$ \\
\hline Article Info \\
\hline $\begin{array}{l}\text { Accepted: } \\
\text { 12 January } 2019 \\
\text { Available Online: } \\
\text { 10 February 2019 }\end{array}$ \\
\hline
\end{tabular}

Wild edible plants (WEPs) are the species that are neither cultivated nor domesticated, but available from their natural habitat and used as a source of food. Use of wild edibles is diminishing at fast pace but it is clear that in many parts of the world the use of wild edibles is still prevalent. Consumption of wild edibles is a major source of vitamins and micro-nutrients for people using only vegetarian diets rich in carbohydrates. The present study deals with the study of diversity and seasonal availability of WEPs and documented 147 species of wild edible plants belonging to 66 families and it reveals information about various edible parts of different species which includes corms, tubers, leaves, flowers, fruits and seeds, which are consumed by tribes and local people. All these plants are common in rural and forest area of Vidarbha region of Maharashtra. Majority of thespecies belongs to the families are Caesalpiniaceae, Fabaceae, Amaranthaceae, Araceae, Rubiaceae, Convolvulaceae, Cucurbitaceae, Dioscoreaceae, Tiliaceae, Anacardiaceae, Bignoniaceae, Combertaceae, Euphorbiaceae, Malvaceae, Moraceae. Collection and utilization of wild edible plants provide seasonal food security and become source of income to the tribes and local people.

\section{Introduction}

Wild edible plants (WEPs) refer to species that are neither cultivated nor domesticated, but available from their natural habitat and used as sources of food (Beluhan and Ranogajec, 2010). Wild edible plants have since ancient times, played a very important role in human life; they have been used for food, medicines, fibre and other purposes and also as fodder for domestic animals (Kanchan, 2011). Various studies have found wild edible plants are the potential source of nutrition while in many cases is more nutritious than the conventionally eaten crops (Grivetti and Ogle, 2000). India is the second largest human populations in this planet, $68.8 \%$ of the population is living in the rural areas (Census, 2011). Most rural communities depend on the resources which are available in nature, including wild edible plants to meet their food needs in periods of food crises, in addition to added food supplements (Rashid, 2008). 
Food insecurity, on the other hand, is a situation of "limited or uncertain availability of nutritionally adequate and safe foods or limited or uncertain ability to acquire acceptable foods in socially acceptable ways", according to the United States Department of Agriculture (USDA). Attaining food security is a matter of prime importance for India where more than one third of its population is estimated to be absolutely poor, and as many as one half of its children have suffered from malnourishment over the last three decades (Ittyerah, 2013).

WEPs are gathered for food, nutrition and livelihoods by different cultures around the world. These plants are gathered from varied habitats from natural forests, agricultural fields to human disturbed areas such as roadsides and wastelands. Forest forms the most important source of wild foods for rural households and forest inhabitants. Among some indigenous people utilization of WEPs is integral component of their culture. Various studies have found wild edible plants are potential source of nutrition while in many cases are more nutritious than conventionally eaten crops (Grivetti and Ogle, 2000). Besides food and nutrition, utilization of WEPs as coping strategies during scarcity is prevalent, particularly in developing countries where food insecurity is more acute. Diversity of plant foods consumed provides nutritional diversity and also food during famine or scarcity of favoured foods (Hatloy et al., 1998). Potential of WEPs in providing source of income and livelihoods in rural settings is acknowledged around the world (Dutta, 2012).

Traditional knowledge exists worldwide in all communities covering varied areas including health, agriculture and natural resource management. In case of the developing world Africa and Latin America are also rich in traditional knowledge but they are to be found by and large only as oral traditions. Asia in general and India in particular have a distinction that traditional knowledge is found not just as oral tradition but also as classical literature that is written down with its own theoretical framework and with a clear exposition of the basic principles of world views (Nene, 2012). There are reports on wild food plants used during famine in Indian conditions (Nene, 2004; Balkundi, 1998). In some areas local food produce is hardly sufficient to meet about two thirds of local requirements. Thus for about four months in each year some of its unfortunate inhabitants have to migrate to the neighbouring cities and remaining people are obliged to subsist on a starvation diet.

These people use many plants, occurring naturally in neighbouring forests, as supplementary food which alone enables them to carry on with their half-starved existence. For that we should document the existing wild edible, which in turn helps in other people could get to know about the edible plants around them. Earlier work on wild edible plants from many parts of Maharashtra like Nasik, Amravati, Buldhana, Kolhapur, Chandrapur, Jalgaon, Akola and Nagpurwere carried out by Patil and Patil, (2000), Bhogaonkar et al., (2010), Kshirsagar et al., (2012), Mahadkar and Jadhav (2013), Joshi et al.,(2013), Reddy, (2012), Patil and Patil, (2012), Jadhav et al., (2011), Patil and Tale, (2018), Thakre and Srinivasu (2012) and Kamble et al., (2013).

\section{Materials and Methods}

The present study was conducted in Vidarbha region of Maharashtra, to identify and document the wild edible plants and their habit, habitat, local availability, season of availability etc. for the period of 2015-16. A gist of Vidarbha region is given below to know about the region. 


\section{Study region}

Vidarbha is the eastern region of the Indian state of Maharashtra, comprising Nagpur Division and Amravati Division (Plate 1). It occupies $31.6 \%$ of the total area and holds $21.3 \%$ of the total population of Maharashtra. Vidarbha is located right at the heart of the Indian Union occupying $97,426 \mathrm{sq}$. $\mathrm{km}$. area between $19^{\circ} 05^{\prime}$ to $21^{\circ} 47^{\prime}$ North latitude and $75^{\circ} 59^{\prime}$ to $79^{\circ} 11^{\prime}$ East longitude. Vidarbha shares its boundary with the state of Madhya Pradesh towards the north, Chattisgarh in the east, Andhra Pradesh in the south and Nasik and Aurangabad administrative division of Maharashtra state in the west. Geographically, Vidarbha lies in the fertile plateau between Melghat in the Satpura range in the north and Balaghat in the Ajantha Range in the south. This area is also known as Payanghat. Rich with bounties of nature Vidarbha consists of the Amravati and Nagpur administrative division of Maharashtra. Amravti division comprises of five districts - Buldhanana, Akola, Washim, Yamatwal besides Amravati district. The Nagpur division comprises of six districts - Wardha, Bhandara, Gondia, Chandrapur, Gardchiroli besides Nagpur district. In all, Vidarbha represents eleven districts. It is the rich biodiversity and varying geographical conditions have been ideal for growth of variety of plants of Vidarbha. Broadly the vegetation can be divided into evergreen forests, deciduous forests and thorny scrub forests, makes it all season availability of wild edible plant resources in this region.

\section{Field survey conducted for documentation of WEPs}

Field survey was conducted through interview, using questionnaire and focal group discussions were made involving traditional medicine practitioner, forest department people and old knowledgeable persons. Interactions with the tribal people about the collection and uses of WEPs from the forest. Special efforts were made to involve women in the exercise, self-help groups (SHG) of women help a lot to document the WEPs. Identification of WEPs is by their local name and availability of different wild edible plants and collection of different parts in different months of the year. All the available information was recorded and a documentation of WEPs was done in required format and analysed using ExcelStat.

\section{Results and Discussion}

\section{WEP resource of Vidarbha region}

The present study documented 147 species of wild edible plants belonging to 66 families. As other studies like, Reddy et al., (2012) documented 61 species of WEPs in Chandrapur district, Deshpande et al., (2015) recorded 73 WEPs belonging to 42 families which are used by Rajgond tribes of Vidarbha region, Patil and Patil (2012) mentioned in his work that there are 43 endangered WEPs are there in Jalgaon district, Bhogaonkaret al., (2010) documented 42 WEPs belonging to 23 families in Melghat forest area of Amravti district and Patil and Tale (2018) documented 20 WEPs used by local people in Akola district.

The detailed analysis of their taxonomic group revealed 147 species, belong to 66 families (Table 1). With respect to families, Caesalpiniaceae shared the largest proportion by consisting of 10 species and followed by Amaranthaceae and Fabaceae with 8 species, Araceae and Rubiaceae with 6 species, Cucurbitaceae and Tiliaceae with 5 species, Anacardiaceae, Combretaceae, Convolvulaceae, Malvaceae, Mimosaceae and Rhamnaceae 4 species each, Dioscoreaceae and Euphorbiaceae with 3 species, these are 
the top fifteen families having highest number of WEP species.

WEPs are available in different life forms. In the present study shows that the largest proportion of wild edible species were in the form of tree i.e.48 species (32.7\%), followed by herbs 44 species (29.9\%), shrubs 28 species (19\%), climbers 23 species (15.6\%), palm 2 species (1.4\%), Aquatic plant and grass one species each $(0.7 \%)$ respectively which is illustrated in the figure 1 . The major consumed wild edible plant part is fruits $24 \%$ and leaves $23 \%$, then followed by whole plant and flowers with $10 \%$ each, seeds $8 \%$, pods $7 \%$, tuber $5 \%$, root $4 \%$, shoot $3 \%$, gum and bulbs with $2 \%$ each, bark $1.4 \%$, stem $0.68 \%$, which is illustrated in the figure 2 . The tribes and local village people consume wild tubers, rhizomes and corms either in raw or baked or boiled or roasted form. Some of the edible parts like, leaves and fruits are also eaten raw, other parts like, tender shoots, flowers, leaves, pods, fruits, etc. and whole plant which are used as vegetable and as well as medicinal purposes. Efforts were also made to explore the nutritive potential of some wild edible tubers, rhizomes, leafy vegetables and wild fruits which supplement several nutrients particularly calcium and carotenoids. Such unconventional wild edible plants are sources of fats, proteins, rich source of micronutrients and trace elements (Nilegaonkar et al., 1985; Kulkarni et al., 2003; Kulkarni, 2006).

\section{Seasonal availability and Occurrence of WEPs in different localities}

In the Vidarbha region, the availability of WEPs is seen all over the year (Figure 3). The peak season of available of WEPs is in the rainy season (June to October) in the different habitats. Majority of the WEPs are herbaceous and short duration climbers are available for human consumption. The reason for the availability of WEPs throughout the year is, one or the other species of wild edible plants show their presence in different seasons and producing different edible parts from them, for example, Annona squamosa produces fruit from December to May, Mangifera indica produces fruit from March to June, Syzygium cumunii produces fruit from June to August, Terminalia catappa produces fruit from August to October, Limonia acidissima produces fruit from November to December, likewise one or other edible parts are available throughout the year. Besides providing good source of nutrients available in different seasons of the year, these species also provide ecological security as they are disease resistant, grow in diverse climates and habitat and ensure sufficient production even in adverse conditions. The production potential of different species and sustainable harvest of useful parts can boost the local economy. Similar type of study was done by Deshpande et al., (2015).

The occurrence of WEPs in Vidarbha region was classified into 11 different localities. The representation of species were maximum in forest area $(70 \%)$, followed by waste land (21 $\%)$, village (15\%), road side and kitchen garden $14 \%$ each, field boundaries (12\%), water body (10\%), marshy area and pond boundaries $5 \%$ each, agriculture field and densely forest area $3 \%$ each, which is illustrated in the figure 4 . Forest is the home for the majority of WEPs. In the Vidarbha region waste lands and road side places are also contributing for food source by the presence of edible plants species there. Most of the villagers and tribal people, in their kitchen garden they grow vegetables, tubers, beans and chillies, etc. (Kulkarni and Kumbhojkar, 1993). 
Table.1 Potential WEPs diversity documented in Vidarbha region

\begin{tabular}{|c|c|c|c|c|c|c|c|}
\hline $\begin{array}{l}\text { Sl. } \\
\text { No. }\end{array}$ & Species & Family & Local name & Habit & Habitat & Season & $\begin{array}{c}\text { Edible } \\
\text { parts }\end{array}$ \\
\hline 1 & $\begin{array}{l}\text { Abelmoschus ficulneus (L.) } \\
\text { Wight \&Arn. }\end{array}$ & Malvaceae & Kasturi & Herb & 2 & Sept - Mar & WP \\
\hline 2 & $\begin{array}{l}\text { Abelmoschus manihot (L.) } \\
\text { Medik }\end{array}$ & Malvaceae & Ran bhendi & Herb & 2,6 & Sept - Feb & $\begin{array}{l}\text { B, R, } \\
\text { Fl., L }\end{array}$ \\
\hline 3 & Abrus precatorius $\mathrm{L}$. & Fabaceae & Gunj & Climber & 11 & Oct - May & L, Fl. \\
\hline 4 & Acacia concinna (Willd.) DC. & Mimosaceae & Shikakai & Climber & $1,3,2$ & Sept - Dec & $\begin{array}{l}\text { Sh., P, } \\
\text { L }\end{array}$ \\
\hline 5 & Acacia nilotica (L.) Willd. & Mimosaceae & Baval, Babool & Tree & $\begin{array}{c}1,4,9,3 \\
2\end{array}$ & TOY & $\begin{array}{l}\text { B, P, } \\
\text { G, S, L }\end{array}$ \\
\hline 6 & Acacia Senegal (L.) Willd. & Mimosaceae & $\begin{array}{l}\text { Goradiyo, } \\
\text { Baval }\end{array}$ & Tree & $1,4,2,3$ & TOY & $\mathrm{L}, \mathrm{G}, \mathrm{P}$ \\
\hline 7 & Aegle marmelos L. Correa & Rutaceae & Bel & Tree & 11 & Mar-June & Fr. \\
\hline 8 & Agave vera-cruz Mill. & Agavaceae & Ghaypat & Shrub & $2,4,1$ & TOY & Fl. \\
\hline 9 & $\begin{array}{l}\text { Alangium salviifolium (L.f.) } \\
\text { Wangerin }\end{array}$ & Alanginaceae & Ankol & Tree & 6 & Mar - Oct & Fr. \\
\hline 10 & $\begin{array}{l}\text { Alocasia macrorhiza (L.) } \\
\text { G.Don. }\end{array}$ & Araceae & Dhopa & Herb & 7,3 & TOY & $\mathrm{L}, \mathrm{Rh}$. \\
\hline 11 & Amaranthus cruentus L. & Amaranthaceae & $\begin{array}{l}\text { Rajgira, } \\
\text { Tandulga }\end{array}$ & Herb & 5 & June - Oct & WP \\
\hline 12 & Amaranthus paniculatus $\mathrm{L}$. & Amaranthaceae & Rajgira & Herb & 5,1 & TOY & WP \\
\hline 13 & Amaranthus tricolour $\mathrm{L}$. & Amaranthaceae & $\begin{array}{l}\text { Taduljira, Lal } \\
\text { mat }\end{array}$ & Herb & 5 & June - Feb & WP \\
\hline 14 & Amarantus spinosus $\mathrm{L}$. & Amaranthaceae & Kale-math & Herb & 2,1 & TOY & $\mathrm{L}, \mathrm{R}$ \\
\hline 15 & Amarantus viridis $\mathrm{L}$. & Amaranthaceae & Tanduliya & Herb & 5,1 & TOY & WP \\
\hline 16 & $\begin{array}{l}\text { Amarphophallus } \\
\text { paeonifollius(Densst) } \\
\text { Nicolson }\end{array}$ & Araceae & Gavathisuran & Shrub & 1,5 & June - Oct & $\mathrm{T}$ \\
\hline 17 & Ammannia baccifera Linn. & Lythraceae & Aginbuti & Herb & 7,8 & June - Oct & $\mathrm{L}$ \\
\hline 18 & $\begin{array}{l}\text { Amorphophallus } \\
\text { campanulatus(Roxb.) Blume }\end{array}$ & Araceae & Suran & Shrub & 1,5 & June - Oct & Rh. \\
\hline 19 & $\begin{array}{l}\text { Amorphophallus } \\
\text { konkanensisHett. }\end{array}$ & Araceae & Bhasmakanda & Herb & 1 & June - Oct & $\mathrm{L}, \mathrm{T}$ \\
\hline 20 & $\begin{array}{l}\text { Andrographis } \\
\text { paniculataWall. }\end{array}$ & Acanthaceae & Kadukirayata & Herb & 11 & June - Feb & WP \\
\hline 21 & Anethum graveolensL. & Apiaceae & Shepu & Herb & 5 & TOY & WP \\
\hline 22 & Annona reticulataL. & Annonaceae & Sitafal & Tree & 4,3 & Dec-May & Fr. \\
\hline 23 & Annona squamosaL. & Annonaceae & Sitafal & Tree & $1,4,3$ & Dec-May & Fr. \\
\hline 24 & $\begin{array}{l}\text { Anogeissus latifolia (DC.) } \\
\text { Wallich }\end{array}$ & Combertaceae & Dhawda & Tree & 1 & Apr - Dec & G \\
\hline 25 & Aristolochia indica $\mathrm{L}$. & Aristolocaceae & Ishwari & Climber & 1 & June - Feb & Fl. \\
\hline 26 & Asparagus racemosus Willd. & Asparagaceae & Shatavari & Herb & 2,1 & June - Jan & WP \\
\hline 27 & Atriplex hortensis L. & Amaranthaceae & Chandanbatva & Shrub & 1 & TOY & WP \\
\hline
\end{tabular}




\begin{tabular}{|c|c|c|c|c|c|c|c|}
\hline 28 & Azadiracta indicaA. Juss. & Meliaceae & Nimboni & Tree & $1,4,3$ & Mar-May & Fr. $\mathrm{S}$ \\
\hline 29 & Basella rubraLinn. & Basellaceae & Bacchalikoora & Climber & 5 & TOY & $\mathrm{L}$ \\
\hline 30 & Bauhinia perpureaL. & Caesalpiniaceae & Kanchan & Tree & 1,5 & Jan - Mar & L, Fl. \\
\hline 31 & Bauhinia racemosaL. & Caesalpiniaceae & Apta & Tree & 1,6 & Mar - July & $\begin{array}{l}\text { L, Sh., } \\
\text { Fl., P }\end{array}$ \\
\hline 32 & Bauhinia vahlii Wight \&Arn. & Caesalpiniaceae & Chamul & Tree & 1 & Apr - Oct & $\mathrm{P}, \mathrm{S}$ \\
\hline 33 & Bauhinia variegate $\mathrm{L}$. & Caesalpiniaceae & $\begin{array}{l}\text { Kanchan, } \\
\text { Kanaraj }\end{array}$ & Tree & $1,6,2$ & Nov - Mar & $\underset{\text { L }}{\text { Sh., Fl. }}$ \\
\hline 34 & Begonia crenataDryand. & Begoniaceae & $\begin{array}{l}\text { Kapru, } \\
\text { Khadak- } \\
\text { ambadi }\end{array}$ & Herb & 9,7 & Aug - Nov & $\mathrm{L}$ \\
\hline 35 & Boerhavia diffusa $\mathrm{L}$. & Nyctaginaceae & Ghetuli & Herb & $4,1,2$ & Aug - Dec & WP \\
\hline 36 & Bombax ceiba $\mathrm{L}$. & Bombacaceae & Katesavar & Tree & 1,3 & Feb - Apr & L, Fl. \\
\hline 37 & Borassus falbellifer Linn. & Arecaceae & Tadi & Palm & $1,4,6,2$ & Nov-May & Fr. \\
\hline 38 & Boswellia serrata Roxb. & Burseraceae & Dhupali, Salai & Tree & 1 & Feb - Jun & $\begin{array}{l}\text { Fr., Fl., } \\
\quad \text { S }\end{array}$ \\
\hline 39 & Brassica compestris Linn. & Crucifereae & $\begin{array}{l}\text { Mustard, Kali } \\
\text { Mohari }\end{array}$ & Herb & 3 & Nov - Apr & $\mathrm{P}$ \\
\hline 40 & Bridelia retusa (L.) A.Juss. & Euphorbiaceae & Kakai, Asana & Tree & 1,11 & TOY & $\mathrm{R}$ \\
\hline 41 & Buchanania lanzan Spreng. & Anacardiaceae & Charoli & Tree & 1 & Apr - May & Fr., $\mathrm{S}$ \\
\hline 42 & $\begin{array}{l}\text { Cannavalia gladiate (Jacq.) } \\
\text { DC. }\end{array}$ & Fabaceae & $\begin{array}{l}\text { Chemmakaay } \\
\text { a }\end{array}$ & Climber & 1,5 & Apr - Oct & Fr. \\
\hline 43 & $\begin{array}{l}\text { Canthium coromandelicum } \\
\text { (N.Burm.) Alst. }\end{array}$ & Rubiaceae & Karbit & Shrub & 1 & Mar - Oct & Fr. \\
\hline 44 & Canthium parviflorum Lamk & Rubiaceae & Katbor & Shrub & 1,4 & Apr - Aug & Fr. L \\
\hline 45 & Capparis zeylanica $\mathrm{L}$. & Capparaceae & $\begin{array}{l}\text { Vaghati, } \\
\text { Orkali }\end{array}$ & Climber & 1 & Mar-May & Fr. \\
\hline 46 & Cassia fistula $\mathrm{L}$. & Caesalpiniaceae & Bahava & Tree & $1,6,4$ & May - Jun & Fl., $\mathrm{P}$ \\
\hline 47 & Cassia mimoesides L. & Caesalpiniaceae & Lajari & Herb & 1 & Aug - Dec & $\mathrm{P}$ \\
\hline 48 & Cassia occidentalis Linn. & Caesalpiniaceae & Marha, Bacca & Shrub & 1 & Aug - Dec & WP \\
\hline 49 & Cassia tora $\mathrm{L}$. & Caesalpiniaceae & Takala, Tarota & Shrub & $2,3,1$ & Aug - Dec & $\begin{array}{l}\text { Sh., L, } \\
\text { Fl., P, } \\
\text { S }\end{array}$ \\
\hline 50 & Celosia argentia $\mathrm{L}$. & Amaranthaceae & Kurdu & Herb & 8 & June - Feb & $\mathrm{L}$ \\
\hline 51 & Ceropegia bulbosa Roxb & Asclepiadaceae & Suparikanda & Climber & 1 & June - Feb & $\mathrm{T}$ \\
\hline 52 & Chenopodium album $\mathrm{L}$. & Chenopodiaceae & Chakvath & Herb & 1,2 & TOY & Sh., L \\
\hline 53 & $\begin{array}{l}\text { Chlorophytum } \\
\text { tuberosum(Roxb.) Baker }\end{array}$ & Liliaceae & $\begin{array}{l}\text { Shevalikardi, } \\
\text { Longdabhaji }\end{array}$ & Herb & 1 & Jun - Sept & $\mathrm{T}, \mathrm{L}$ \\
\hline 54 & $\begin{array}{l}\text { Citrullus colocynthis }(\mathrm{L} .) \\
\text { Schrad. }\end{array}$ & Cucurbitaceae & $\begin{array}{l}\text { Kadu- } \\
\text { indravani }\end{array}$ & Climber & 7,2 & May - Oct & WP \\
\hline 55 & $\begin{array}{l}\text { Clerodendrum seratum } \mathrm{L} \text {. } \\
\text { Moon }\end{array}$ & Verbenaceae & Bharangi & Shrub & $1,4,6$ & Oct-May & L, Fl. \\
\hline 56 & Coccinia grandis(L.) Voigt. & Cucurbitaceae & Tondali & Climber & 3,1 & Dec - Apr & Fr. \\
\hline 57 & Colocasia esculentaL. & Araceae & Kochai & Herb & $5,8,7,9$ & Sept - Nov & $\mathrm{L}, \mathrm{T}$ \\
\hline 58 & Commelina benghalensisL. & Commelinaceae & Kena & Herb & 4,7 & June - Oct & $\mathrm{L}$ \\
\hline 59 & Corchorus capsularis L & Tiliaceae & Chunch & Herb & 3 & Mar-May & $\mathrm{L}$ \\
\hline
\end{tabular}




\begin{tabular}{|c|c|c|c|c|c|c|c|}
\hline 60 & Cordia dichotomaForst. & Boraginaceae & Bhokar & Tree & 1,7 & Nov - Feb & Fr. \\
\hline 61 & $\begin{array}{l}\text { Costus speciosus(Koen) J.E. } \\
\text { Smith. }\end{array}$ & Zingiberaceae & Harduli & Herb & 1 & June - Oct & $\mathrm{T}$ \\
\hline 62 & Crotalaria junceaL. & Fabaceae & Boru & Shrub & 1,10 & July - Sept & $\mathrm{L}, \mathrm{S}$ \\
\hline 63 & Curculigo orchioides Gaertn. & Hypoxidaceae & Kali Musali & Herb & 1 & June - Oct & $\mathrm{T}$ \\
\hline 64 & $\begin{array}{l}\text { Dendrocalamus } \\
\text { strictus(Roxb.) Nees. }\end{array}$ & Poaceae & Vaste & Grass & 1 & June - Oct & Sh. \\
\hline 65 & Dentella repens(L.) J.\& G. & Rubiaceae & Kadubhaji & Herb & 8 & June - Feb & $\mathrm{L}$ \\
\hline 66 & Digeramuricata(L.) Mart. & Amaranthaceae & Gitana, Getna & Herb & $5,2,1$ & June - Oct & $\mathrm{L}$ \\
\hline 67 & $\begin{array}{l}\text { Dioscorea belophylla(Prain) } \\
\text { Voigt. }\end{array}$ & Dioscoreaceae & Kadu-karand & Climber & 1,5 & TOY & $\mathrm{T}$ \\
\hline 68 & DioscoreabulbiferaL. & Dioscoreaceae & Dukkar-kand & Climber & 1,5 & Sept - Oct & T, Fl. \\
\hline 69 & DioscoriapentaphyllaL. & Dioscoreaceae & Shendvel & Climber & 1,5 & July - Sept & Fl., $\mathrm{T}$ \\
\hline 70 & Diospyrosmelanoxylon Roxb. & Ebenaceae & Tembhurni & Tree & 1,11 & Mar-Sept & $\begin{array}{l}\text { Fr., R, } \\
\mathrm{S}\end{array}$ \\
\hline 71 & $\begin{array}{l}\text { Diplocyclospalmatus(L.) } \\
\text { C.Jeffrey }\end{array}$ & Cucurbitaceae & $\begin{array}{l}\text { Ghungarubhaj } \\
\text { i, Shivling }\end{array}$ & Climber & 1,3 & Aug - Feb & WP \\
\hline 72 & Drimiaindica (Roxb.) Jessop & Liliaceae & Rankanda & Herb & 1 & MS & $\mathrm{B}, \mathrm{L}$ \\
\hline 73 & EmblicaofficinalisGaertn & Euphorbiaceae & Awala. & Tree & 1 & Nov - Feb & Fr. \\
\hline 74 & Emilia sonchifolia(Linn.) DC. & Asteraceae & Makka & Herb & $10,2,6$ & TOY & L, Sh. \\
\hline 75 & $\begin{array}{l}\text { Ensetesuperbum(Roxb.) } \\
\text { Cheesuran }\end{array}$ & Musaceae & $\begin{array}{l}\text { Chaveni, } \\
\text { Raankel }\end{array}$ & Herb & 1,6 & TOY & $\begin{array}{l}\text { R, L, } \\
\text { Fl., Fr. }\end{array}$ \\
\hline 76 & FicusbenghalensisL. & Moraceae & Wad & Tree & $1,3,2,4$ & TOY & $\mathrm{B}, \mathrm{L}$ \\
\hline 77 & FicusracemosaL. & Moraceae & Umbar & Tree & $2,3,4,1$ & Feb - July & Fr. \\
\hline 78 & FicusreligiosaL. & Moraceae & Pimpal & Tree & $1,4,2,3$ & TOY & $\mathrm{L}$ \\
\hline 79 & $\begin{array}{l}\text { Flacourtiaindica(Burm.f.) } \\
\text { Merr. }\end{array}$ & Flacaurtiaceae & Karai & Shrub & 1 & Dec-July & Fr. \\
\hline 80 & $\begin{array}{l}\text { Glycosmispentaphylla(Retz) } \\
\text { DC. }\end{array}$ & Rutaceae & Ban Nimbu & Climber & 1 & Nov - May & Fr. \\
\hline 81 & GrewiaabutilifoliaW. Vent. & Tiliaceae & $\begin{array}{l}\text { Vern, Tel } \\
\text { Chhopdi }\end{array}$ & Shrub & 1,6 & May - Oct & Fr. \\
\hline 82 & GrewiaasiaticaL. & Tiliaceae & Goyalipala & Shrub & 1 & $\mathrm{Feb}-\mathrm{Mar}$ & Fr. \\
\hline 83 & GrewiasclerophyllaRoxb. & Tiliaceae. & Joddhaman & Shrub & 1 & Sept - Jan & Fr. \\
\hline 84 & GrewiatilifoliaVahl. & Tiliaceae & Dhaman & Tree & 1 & May-Sept & Fr. \\
\hline 85 & Hibiscus cannabinusL. & Malvaceae & Aambadi & Shrub & 5,3 & TOY & $\begin{array}{l}\text { L, Fl., } \\
\text { S }\end{array}$ \\
\hline 86 & Holarrhenapubescens Wall. & Apocynaceae & Pandhrakuda & Shrub & 1 & Nov - Feb & $\begin{array}{l}\text { Fl., P, } \\
\text { L, S }\end{array}$ \\
\hline 87 & IndigoferaglandulosaWendl. & Fabaceae & Barbath & Herb & 1,10 & June - Feb & $\mathrm{L}$ \\
\hline 88 & Ipomoea aquatcaForssk. & Convolvulaceae & Karembua & Climber & $8,7,9,3$ & June - Feb & WP \\
\hline 89 & Ipomoea muricata(L.)Jacq. & Convolvulaceae & Bhovara & Climber & $8,7,9,3$ & June - Feb & Fr. \\
\hline 90 & Ipomoea turbinataLag. & Convolvulaceae & Gariya & Climber & $8,7,9,3$ & June - Feb & Fl. \\
\hline 91 & $\begin{array}{l}\text { Lanneacoromandelica(Houtt.) } \\
\text { Merr. }\end{array}$ & Anacardiaceae & Moi, Shemat & Tree & 1 & Feb - Apr & L, Fr. \\
\hline 92 & Lantana camaraL. & Verbanaceae & Ghaneri & Shrub & $1,2,3,4$ & TOY & Fr. L \\
\hline 93 & Leeaasiatica(L.) Ridsdale & Leeaceae & Deenda & Shrub & 1 & Mar-Nov & $\mathrm{L}$ \\
\hline
\end{tabular}




\begin{tabular}{|c|c|c|c|c|c|c|c|}
\hline 94 & LimoniaacidissimaL. & Rutaceae & $\begin{array}{l}\text { Kavanth, } \\
\text { Kaithbael }\end{array}$ & Tree & 10 & Nov - Dec & Fr. \\
\hline 95 & $\begin{array}{l}\text { Madhucalongifolia(Koen.) } \\
\text { Mac. }\end{array}$ & Sapotaceae & Moha & Tree & 1,9 & Dec-July & Fr. \\
\hline 96 & Maesaindica(Roxb.) DC. & Myrsinaceae & Atki, Arngen & Shrub & 1 & Sept - May & L, Fr. \\
\hline 97 & MangiferaindicaL. & Anacardiaceae & Aam & Tree & 1,5 & Mar-June & L, Fr. \\
\hline 98 & $\begin{array}{l}\text { Manilkarahexandra(Roxb.) } \\
\text { Dub. }\end{array}$ & Sapotaceae & Khirni & Tree & 1 & Dec - Apr & Fr. \\
\hline 99 & MenthaviridisLinn. & Lamiaceae & Pudina, Mint & Herb & $1,3,5$ & TOY & WP \\
\hline 100 & MomordicadioicaRoxb. & Cucurbitaceae & $\begin{array}{l}\text { Katwal, } \\
\text { Kartoli }\end{array}$ & Climber & 6,1 & June - Nov & WP \\
\hline 101 & Morindacitrifolia $\mathrm{L}$. & Rubiaceae & Noni & Shrub & $1,3,5$ & TOY & $\begin{array}{l}\text { R, L, } \\
\text { Fr. }\end{array}$ \\
\hline 102 & MorindapubescensJ.E.Sm. & Rubiaceae & $\begin{array}{l}\text { Bartondi, } \\
\text { Nonifal }\end{array}$ & Tree & 4 & Mar-May & Fr. \\
\hline 103 & MoringaoleiferaLam. & Moringaceace & Shevga & Tree & $5,3,6$ & TOY & $\mathrm{L}, \mathrm{P}, \mathrm{S}$ \\
\hline 104 & Mucunapruriens(L.) DC. & Fabaceae & Khajkoyali. & Climber & 1,7 & June - Oct & $\mathrm{P}$ \\
\hline 105 & NelumbonисiferaGaertn. & Nelumbonaceae & Kamal & Climber & 7,8 & TOY & WP \\
\hline 106 & OlaximbricataRoxb. & Olacaceae & Aratpari & Shrub & 3 & TOY & $\mathrm{L}$ \\
\hline 107 & OpuntiaelatiorMill. & Cactaceae & $\begin{array}{l}\text { Phadyanivadu } \\
\text { ng }\end{array}$ & Shrub & $1,2,6$ & Aug - Jan & Fr. \\
\hline 108 & Oroxylonindicum(L.) Vent. & Bignoniaceae & Tetu & Tree & 1 & Feb - Mar & P, Fl. \\
\hline 109 & OryzarufipogonGriff. & Poaceae & $\begin{array}{l}\text { Devbhat, } \\
\text { Tharsod }\end{array}$ & Herb & 6 & Sept - Jan & $\mathrm{S}$ \\
\hline 110 & Oxalis corniculataL. & Oxalidaceae & $\begin{array}{l}\text { Tinpatti, } \\
\text { Ambushi }\end{array}$ & Herb & 1,8 & TOY & L, Fr. \\
\hline 111 & Oxalis dehradunensisRaiz. & Oxalidaceae & Dun Ambushi & Herb & 1,5 & TOY & $\mathrm{L}$ \\
\hline 112 & Phoenix sylvestris(L.) Roxb. & Arecaceae & Sindi & Palm & $1,6,9$ & Mar-May & Fr. \\
\hline 113 & $\begin{array}{l}\text { PhyllanthusamarusSchum } \\
\text { and Thonn }\end{array}$ & Euphorbiaceae & Bhuiavala & Herb & 2 & June - Feb & Fr. \\
\hline 114 & Physalis minima $\mathrm{L}$. & Solanaceae & $\begin{array}{l}\text { Ran-popti, } \\
\text { chirboti }\end{array}$ & Herb & $2,6,4$ & TOY & Fr. \\
\hline 115 & $\begin{array}{l}\text { Pithecellobiumdulce(Roxb.) } \\
\text { Benth. }\end{array}$ & Mimosaceae & Chichbilai & Tree & 1,3 & June - Oct & $\begin{array}{l}\mathrm{B}, \mathrm{L}, \\
\mathrm{P}, \mathrm{S}\end{array}$ \\
\hline 116 & PlumbagozeylanicaL. & Plumbaginaceae & Chitrak & Shrub & $1,2,9$ & TOY & $\mathrm{L}$ \\
\hline 117 & PortulacaoleraceaLinn. & Portulacaceae & Gholbhaji & Herb & 10 & TOY & WP \\
\hline 118 & PortulacaquadrifidaL. & Portulacaceae & Ranghol & Herb & 2,4 & TOY & WP \\
\hline 119 & PuerariaTosa(Roxb.) DC. & Fabaceae & $\begin{array}{l}\text { Davankanda, } \\
\text { Pithana }\end{array}$ & Climber & 1 & TOY & $\mathrm{T}$ \\
\hline 120 & Rhynchosia minima (L.) DC. & Fabaceae & Chipali. & Climber & 2 & June - Feb & $\mathrm{P}$ \\
\hline 121 & $\begin{array}{l}\text { Riveahypocrateriformis(Desr. } \\
\text { )Choisy. }\end{array}$ & Convolvulaceae & Phaangi & Climber & 6,1 & Dec - Feb & L, Fl. \\
\hline 122 & RumexvesicariusL. & Polygonaceae & Aambadchuka & Herb & 1 & TOY & WP \\
\hline 123 & $\begin{array}{l}\text { Schleicheraoleosa(Lour.) } \\
\text { Oken. }\end{array}$ & Sapindaceae. & Kusumb & Tree & 1 & Mar-June & L, Fr. \\
\hline 124 & Scilla hyacinthine (Roth.) Mc. & Liliaceae & Kapuskanda, & Herb & 1 & June - Oct & $\mathrm{T}$ \\
\hline
\end{tabular}




\begin{tabular}{|c|c|c|c|c|c|c|c|}
\hline & Bride. & & Shakkarkanda & & & & \\
\hline 125 & SemecarpusanacardiumL. & Anacardiaceae & Bibba, Billava & Tree & 1 & May - July & Fr. \\
\hline 126 & $\begin{array}{l}\text { Sennahirsuta }(\mathrm{L} .) \\
\text { H.S.Irwin\&Barneby }\end{array}$ & Caesalpiniaceae & $\begin{array}{l}\text { Karuthagarai, } \\
\text { Thagarai }\end{array}$ & Shrub & 1 & Sept - Dec & $\mathrm{L}$ \\
\hline 127 & Sesbaniagrandiflora (L.) Pers. & Fabaceae & Hatga & Tree & $1,6,2$ & Nov-May & $\begin{array}{l}\text { L, Fl., } \\
\text { P }\end{array}$ \\
\hline 128 & Smilax sp. & Smilacaceae & Ram datum & Shrub & 1 & June - Feb & WP \\
\hline 129 & $\begin{array}{l}\text { Solenaamplexicaulis(Lam.) } \\
\text { Gandhi }\end{array}$ & Cucurbitaceae & Gometi. & Climber & 7 & June - Oct & Fr. \\
\hline 130 & SterculiaurensRoxb. & Malvaceae & $\begin{array}{l}\text { Kawali, } \\
\text { Kandol }\end{array}$ & Tree & 1,2 & TOY & $\mathrm{G}, \mathrm{S}$ \\
\hline 131 & $\begin{array}{l}\text { Syzygiumcumunii(Linn.) } \\
\text { Skeels. }\end{array}$ & Myrtaceae & Jambhul & Tree & $1,3,4$ & June - Aug & Fr. \\
\hline 132 & $\begin{array}{l}\text { Taccaleontopetaloides (L.) O. } \\
\text { Ktze. }\end{array}$ & Taccaceae & $\begin{array}{l}\text { Devkand, } \\
\text { Varade }\end{array}$ & Herb & 1,2 & Apr - Oct & $\mathrm{T}$ \\
\hline 133 & TamarindusindicaLinn. & $\begin{array}{l}\text { Caesalpinnacea } \\
\mathrm{e}\end{array}$ & Chincha & Tree & $1,4,6$ & TOY & WP \\
\hline 134 & $\begin{array}{l}\text { Tamilnadiauliginosa(Retz.) } \\
\text { Triveng and Sastry }\end{array}$ & Rubiaceae & Pendhar. & Tree & 1 & Aug - Mar & Fr. \\
\hline 135 & $\begin{array}{l}\text { Terminaliabellirica (Gaertn.) } \\
\text { Roxb. }\end{array}$ & Combertaceae & Behda & Tree & 1 & Nov - Feb & Fr., $\mathrm{S}$ \\
\hline 136 & TerminaliacatappaL. & Combertaceae & Janglibadam & Tree & 1 & Aug - Oct & $\begin{array}{l}\text { Fr., L, } \\
\text { B }\end{array}$ \\
\hline 137 & TerminaliacuneataRoth. & Combretaceae & Arjun & Tree & 1,7 & Nov - June & Fr. \\
\hline 138 & $\begin{array}{l}\text { Theriophonumindicum(Dalz.) } \\
\text { Engler }\end{array}$ & Araceae & Undirkani & Herb & 1 & June - Oct & L, St. \\
\hline 139 & TrapaNatansLinn. & Trapaceae & Shingada & $\begin{array}{c}\text { Aquatic } \\
\text { Plant }\end{array}$ & 7 & Mar-May & $\mathrm{S}$ \\
\hline 140 & Tricodesmaindicum(L.) Lehm & Boraginaceae & Phopati & Herb & 4 & June - Feb & Fr. \\
\hline 141 & Woodfordiafruticosa(L.)Kurz. & Lythraceae & $\begin{array}{l}\text { Dowari, } \\
\text { Dhayti }\end{array}$ & Shrub & 10,5 & Jan - Apr & $\begin{array}{l}\text { L, Fl., } \\
\text { Fr., G }\end{array}$ \\
\hline 142 & $\begin{array}{l}\text { Wrightiatinctoria(Roxb.) } \\
\text { R.Br. }\end{array}$ & Apocynaceae & Kala Kuda & Tree & 1 & Mar - June & Fl. \\
\hline 143 & Xanthium indicumL. & Asteraceae & Ghagara & Shrub & 2,1 & Nov - Feb & Fr. \\
\hline 144 & $\begin{array}{l}\text { Ziziphusglaberrima(Sedgw.) } \\
\text { Sant. }\end{array}$ & Rhamnaceae & Goti & Tree & 1,2 & Mar-May & Fr. \\
\hline 145 & ZiziphusjujubaMill. & Rhamnaceae & MotaBor & Tree & 1,2 & Oct - Mar & Fr. \\
\hline 146 & Ziziphusmauritiana Lam. & Rhamnaceae & Bor & Tree & 1,2 & Dec-Mar & Fr. \\
\hline 147 & ZizipusoenopliaLinn. Mill & Rhamnaceae & Yeroni & Tree & 1 & Nov - May & Fr. \\
\hline
\end{tabular}

Note: Habitat >1-forest area, 2-waste land, 3-village, 4-road side, 5-kitchen garden, 6-forest boundaries, 7-water bodies, 8marshy area, 9-pond boundaries, 10-agriculture field, 11-densely forest area. Edibleparts > Fr.-fruits, L-leaves, WP-whole plant, Fl.-flower, S-seeds, P-pods, T-tuber, R-root, Sh.-shoot, B-bulb, G-gum, Rh.-rhizome, B-bark, St-stem 
Fig.1 Life form wise Distribution of WEPs in the study region

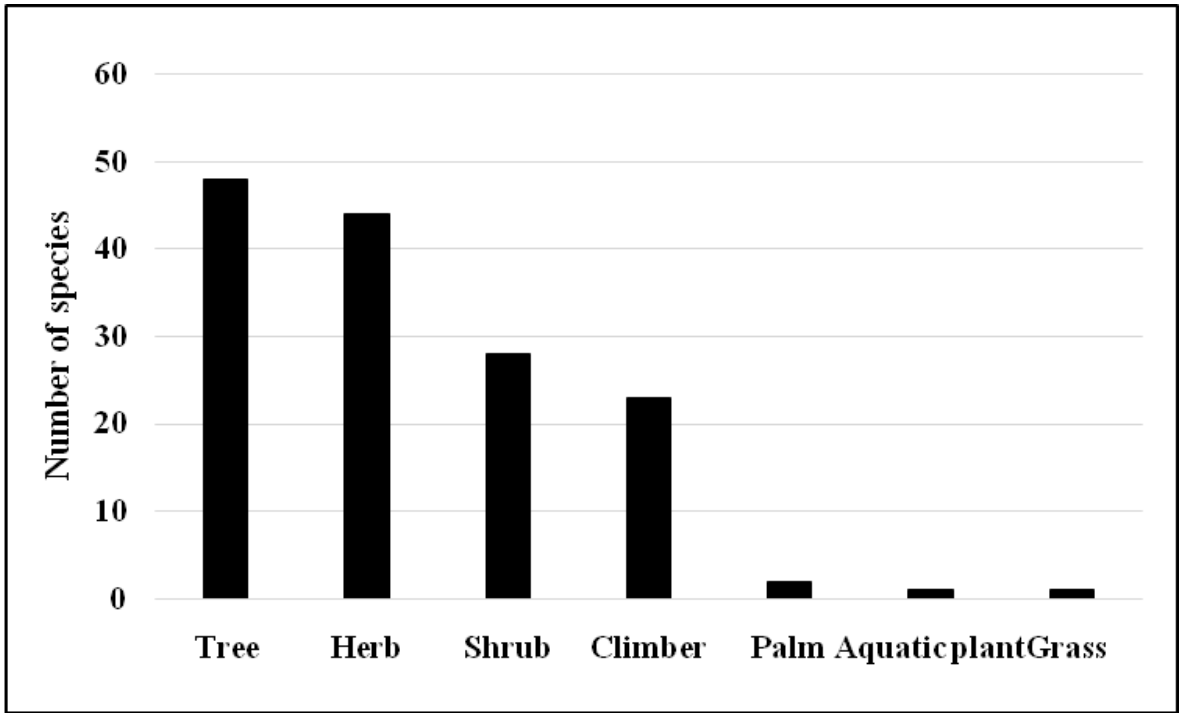

Fig.2 Plant parts used as edible in study region

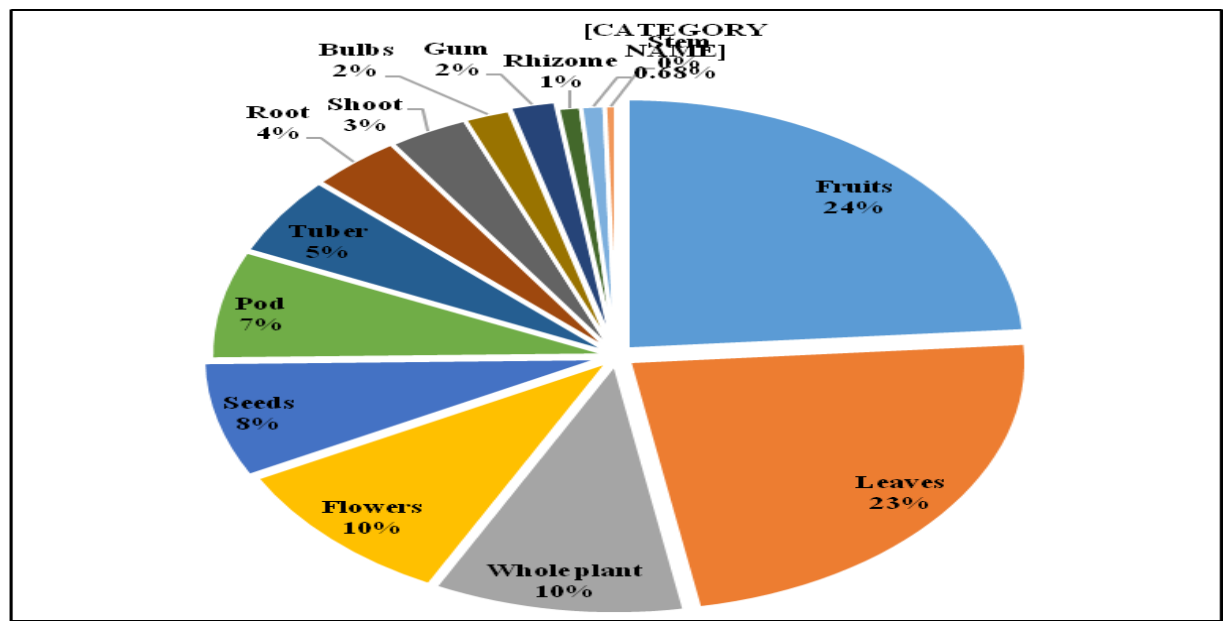

Fig.3 Availability of wild edibles in the study region

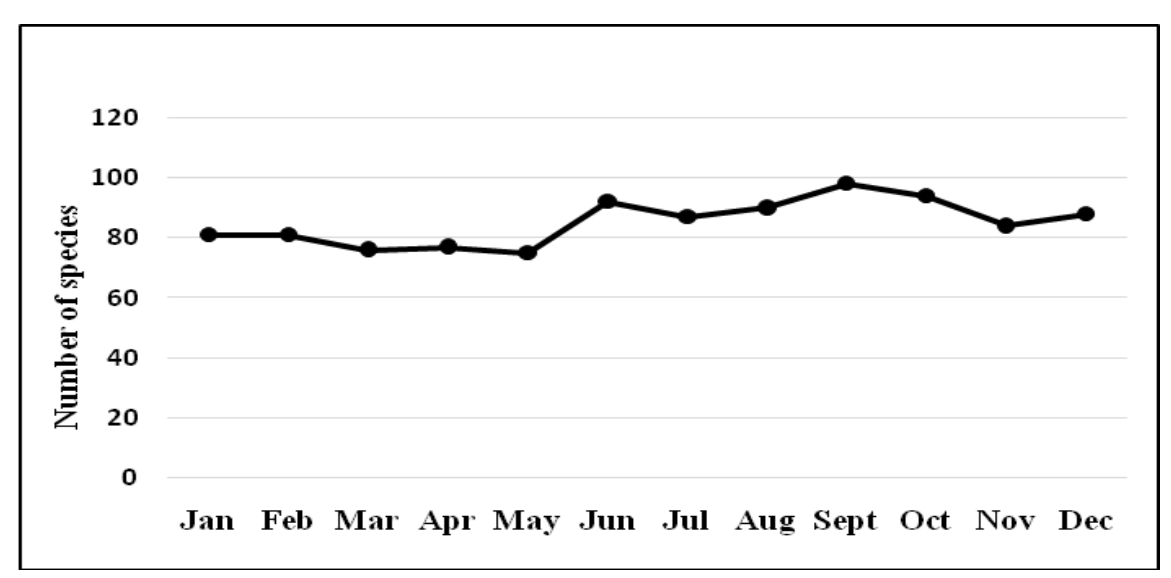


Fig.4 WEPs located in different habitats

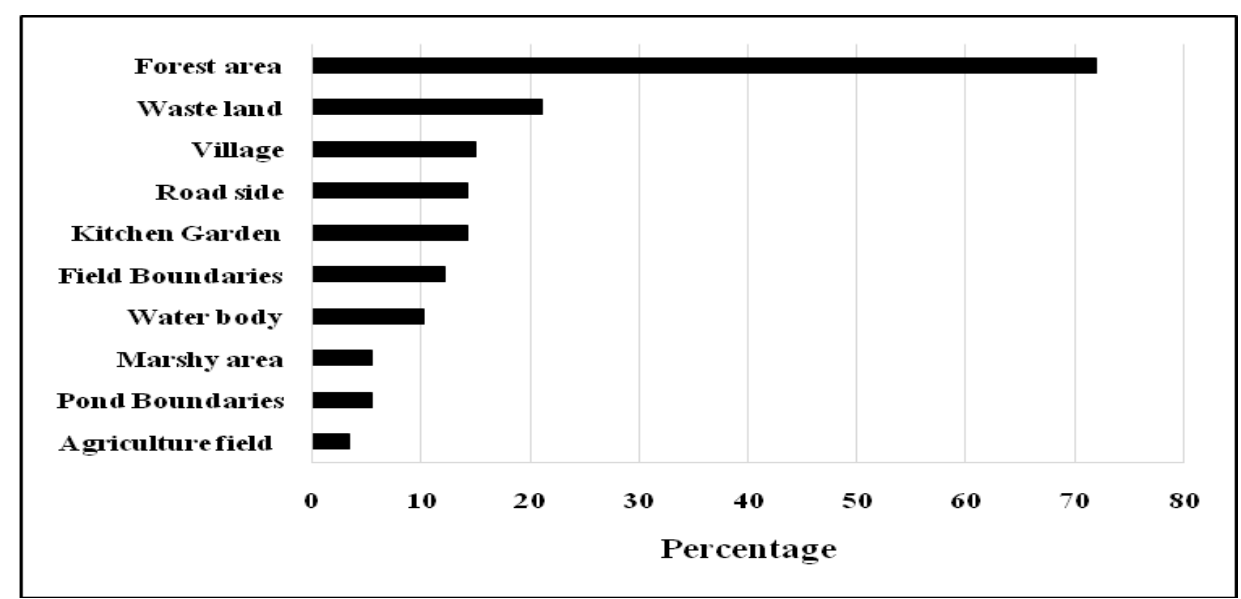

Plate.1 Study area

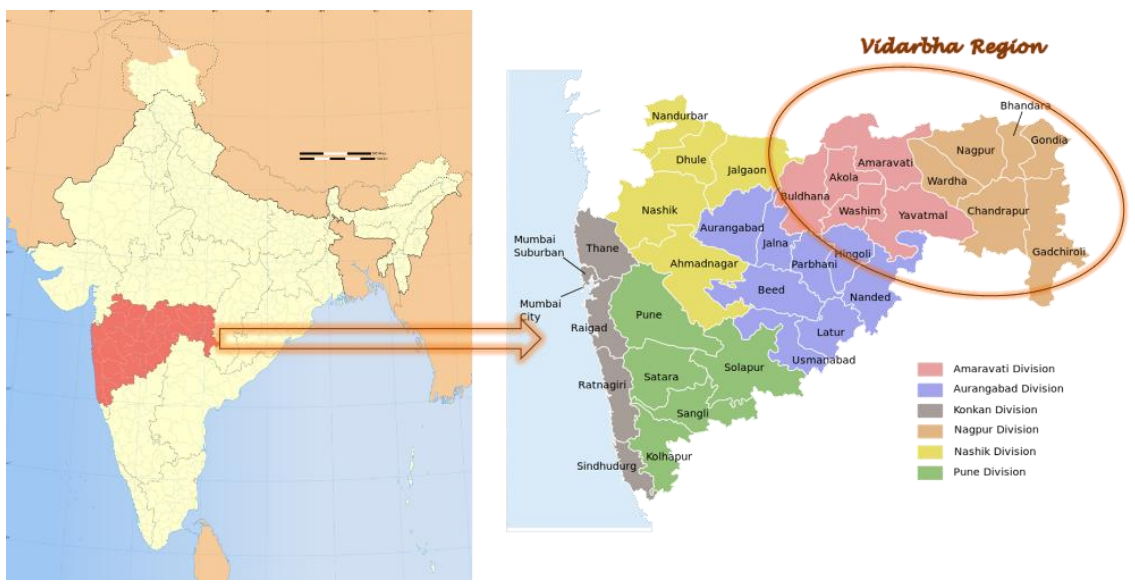

In conclusion, wild species and intra-species biodiversity have key roles in global food security. Use of wild edible vegetables is diminishing at fast pace but it is clear that in many parts of the world the use of wild vegetables is still prevalent. Wild edible plants documentations show their various adaptability to all kinds of ecosystem and seasonal availability. Wild edible plants are available during food crisis period. Wild edible plants are in par with cultivated plants in terms of seasonal food security and nutritional security by availing themselves throughout the year in various forms. Hence, there is a need to preserve traditional knowledge as well as conserve these genetic resources mostly those of wild relatives of crop plants.

\section{Future Line of Study}

Intensive research must be encouraged on Where? When? And How much? of wild edible plants is required for cultivating commercially. Biodiversity questions and/or prompts need to be included in food consumption surveys to evaluate the wild edible plants performance. Documentation of existing wild edible plants and protection of their habitat in the nature. Acquiring nutrient 
data on existing biodiversity needs to be a prerequisite for decision making in GMO work and cultivar promotion. Reforestation work of wild edible plants must be encouraged. Valuation of wild edible plants in different ecosystem. Empowering wild edible plants collection, processing and value addition. Developing technologies for processing and value addition of wild edible plants. There is an urgent need of Food forest to meet the food requirement and also nutrition security for increasing population.

\section{References}

Balkundi, H.V. 1998. Famines and droughts in the Indian subcontinent during the $5^{\text {th }}$ century $\mathrm{BC}$ to $18^{\text {th }}$ Century AD. Asian Agri-History. 2:305-315.

Beluhan, S. and Ranjogajec, A.2010. Chemical composition and nonvolatile components crotial wild edible mushrooms. Food chemistry. 124:1076-1082.

Bhogaonkar, P.Y., V. R.Marathe and Kshirsagar, P.P. 2010. Documentation of wild edible plants of Melghat Forest, Dist. Amaravati, Maharashtra State, India. Ethnobotanical Leaflets. 14:751-758.

Census of India, 2011. Office of the registrar general and census commissioner, India. Ministry of home affairs, Government of India, 1:2. (http://www.censusindia.gov.in/vital_s tatistics/SRS_Reports.html)

Deshpande, S.,R. Joshi and Kulkarni, D.K. 2015. Nutritious Wild Food Resources of Rajgond Tribe, Vidarbha, Maharashtra State, India. Indian Journal of Fundamental and Applied Life Sciences. 5(1):15-25.

Dutta, U. 2012. Wild vegetables collected by the local communities from the Chirang Reserved Forest of BTAD, Assam. International Journal of
Science and Advanced Technology. 2(4):116-126.

Grivetti, L. E. and Ogle, B.M. 2000. Value of traditional foods in meeting macro and micronutrient needs: the wild plant connection. Nutrition Research Reviews. 13:31-46.

Hatloy, A., L.E. Torheim and Oshaug,A. 1998. Food variety- a good indicator of nutritional adequacy of the diet: A case study from an urban area in Mali, West Africa. European Journal of Clinical Nutrition. 52:891-898.

Ittyerah, A. C. 2013. Food security in India: Issues and suggestions for effectiveness. Indian institute of public administration, New Delhi.

Jadhav, V.D., S. D. Mahadkar and Valvi, S.R. 2011. Documentation and ethnobotanical survey of wild edible plants from Kolhapur district. Recent Research in Science and Technology. 3(12):58-63.

Joshi, R., N. Phansalkar, D. K. Kulkarni,A. Chothe, S.Patil, S. Kale, B. Parmar and Ahire M. 2013. Unfolding the potential of tribal food resources of Western India. BAIF Development Research Foundation, Pune, India. Pp. 1-37.

Kamble, R.B., S. Hate and Chaturvedi, A.2013. New additions to the Flora of Nagpur District, Maharashtra. Journal of New Biology Reports. 2(1):09-13.

Kanchan, L. V. 2011. Nutritional analysis of indigenous wild herbs used in eastern Chhattisgarh India. Emir journal of food and agriculture. 23(6):554-560.

Kshirsagar, P.P., V. R. Maratheand Bhogankar, P.Y. 2012. Documentation of wild edible plants of Buldhana district, Maharashtra, India. Life Sciences Leaflets. 5:29-36.

Kulkarni, D.K., V. V. Agteand Kumbhojkar, M.S. 2003. Leafy vegetables consumed by Mahadeokoli tribe in 
Western Maharashtra with their nutritional potential. Ethnobotany. 15:34-38.

Kulkarni, D. K. 2006. Role of ethno-botany in Modern Agriculture. In Proceeding of National Conference on Bridging Gap between Ancient and Modern Technologies to Increase Agricultural Productivity, edited by Chudhary SL, Saxena RC and Nene YL (Pub. Central Arid Zone Research Institute) Jodhpur, Rajasthan, India. Pp. 104115.

Kulkarni, D. K. and Kmbhojkar, M. S. 1993. Kitchen garden plants of Mahadeokoli tribe in Maharashtra. Ethnobotany. 5: 119-127.

Mahadkar, S. and Jadhav, V. 2013. Traditional uses of some wild edible plants from Kolhapur district. Life Sciences Leaflets. 5:19-26.

Nene, Y.L. 2004. Plant species utilized as food during famines and their relevance today. Asian Agri-History. 8(4): 267-278.

Nene, Y.L. 2012. Significant milestones in Evolution of agriculture in the World. Asian Agri-History. 16(3):219-235.

Nilegaonkar, S., V. D. Vartak and Chitre, R. G.1985. Nutritional evaluation of some wild food plants from Pune and neighbouring districts, Maharashtra
state-part-I. Journal of Economic and Taxonomic Botany. 6(3): 629-635.

Patil, J.K., and Patil, S.V. 2012. Biodiversity of vulnerable and endangered plants from Jalgaon district of North Maharashtra. Asian Journal of Pharmacy and Life Science. 2(2):144150.

Patil, M.V., and Patil, D.A. 2000. Some More Wild Edible Plants of Nasik District, Maharashtra. Ancient Science of Life. 19(3/4): 102-104.

Patil, V.S., and Tale, O.S. 2018. Preliminary Survey on Occurrence and Distribution of Wild Vegetables from Akola District. International Journal of Advance Research and Innovative Ideas in Education. 4(1): 16-20.

Rashid, A. 2008. Less known wild edible plants used by the Gujjar tribe of district Rajouri, Jammu \& Kashmir state, India. International journal of Botany, 4(2):219-224.

Reddy, B.M. 2012. Wild edible plants of Chandrapur district, Maharashtra, India. Indian Journal of Natural Products and Resources. 3(1):110-117.

Thakre, M.T., and Srinivasu, T. 2012. New (Fabaceae member) records to Nagpur district. M.F.P. News XXII. (2):4-5.

\section{How to cite this article:}

Kiran, K.C., C. Dhanush, C.V Gajendra and Reddy, B.M. 2019. Diversity and Seasonal Availability of Potential Wild Edible Plants from Vidarbha Region of Maharashtra State, India. Int.J.Curr.Microbiol.App.Sci. 8(02): 1434-1446. doi: https://doi.org/10.20546/ijcmas.2019.802.167 